\title{
TURBO EQUALIZATION OF SERIALLY CONCATENATED SYSTEMATIC CONVOLUTIONAL CODES AND SYSTEMATIC SPACE TIME TRELLIS CODES
}

\author{
B. L. Yeap, T. H. Liew and L. Hanzo \\ Dept. of Electr. and Comp. Sc., Univ. of Southampton, SO17 1BJ, UK. \\ Tel: +44-703-593 125, Fax: +44-703-593 045 \\ Email: lh@ecs.soton.ac.uk \\ http://www-mobile.ecs.soton.ac.uk
}

\begin{abstract}
A novel turbo equalization arrangement is proposed, which is constituted by a serially concatenated systematic convolutional codec and a systematic space time trellis code (CC-SSTTC) assisted scheme. The BER performance of this scheme using various interleaving sizes was characterised over an equal-power twopath Rayleigh fading channel. After eight turbo equalization iterations, an interleaving gain of approximately $5.5 \mathrm{~dB}$ was recorded at $\mathrm{BER}=$ $10^{-4}$ for the CC-SSTTC system using a 6400bit Space Time Trellis (STT) interleaver, when compared to the same CC-SSTTC scheme utilising a 100-bit STT interleaver. It was also observed that the CC-SSTTC scheme employing a 6400-bit STT interleaver outperformed the STT coded system utilising the same STT interleaver but without channel coding by approximately $5.7 \mathrm{~dB}$ at $\mathrm{BER}=10^{-4}$.
\end{abstract}

\section{INTRODUCTION}

The IMT2000 system aims to support full coverage and mobility for a bit rate of at least $144 \mathrm{kbps}$, but preferably 384 at kbps and to provide a limited coverage and mobility for a bit rate of $2 \mathrm{Mbps}$ [1]. The $3 \mathrm{G}$ proposals also aim to provide an improved spectrum efficiency compared to existing systems.

However, there are problems associated with high data rate transmissions, since systems transmitting at high bit rates, such as $2 \mathrm{Mbps}$, experience a high grade of channel-induced dispersion and suffer from Inter Symbol Interference (ISI). Hence, the equalizer design must be capable of mitigating the effects of ISI. In conjunction with equalization, channel decoding can also be employed in order to further improve the performance of the system. Powerful error correction schemes, such as turbo codes [2], have been shown to yield performances close to Shannonian performance limits. The discovery of turbo codes and turbo decoding led to the development of turbo equalization [3]. Turbo equalization is a technique that performs channel equalization and channel decoding jointly and iteratively. This scheme has been shown to successfully mitigate the effects of channel ISI.

Recently a range of transmission diversity techniques known as Space Time Trellis (STT) coding [4] have also been introduced in order to provide diversity gain for Mobile Stations (MSs) by upgrading the Base Stations (BSs). STT coding [4] jointly designs the channel coding, modulation, transmit diversity and the optional receiver diversity. Subsequently, Bauch et al. [5] proposed a joint equalization and STT decoding scheme, which yielded an improved performance due to exploiting the soft-decision based feedback from the STT decoder to the equalizer's input.

Motivated by these trends, in this contribution we aim to improve the performance of the STT encoded system by employing additional channel encoding and turbo equalization. Specifically, we propose a turbo equalization scheme for a system, which serially concatenates a systematic Convolutional Code and a Systematic STT Code (CC-SSTTC). The performance of this system is investigated for various STT interleaver sizes $\pi_{s}$, namely $100,400,800,1600,3200$ and 6400 bits.

The outline of this paper is as follows. Section 2 presents an overview of the investigated system. This is followed by a description of the turbo equalization principle in the context of our serial concatenated scheme in Section 3. Performance results are presented in Section 4 and finally, the main conclusions are summarised in Section 5.

\section{SYSTEM OVERVIEW}

The schematic of the CC-SSTTC transmitter consists of a serially concatenated systematic convolutional code 


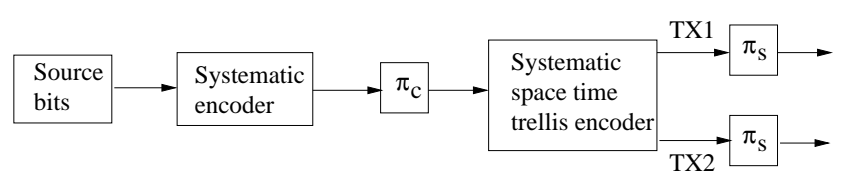

Figure 1: Transmitter of the serially concatenated systematic convolutional coded and systematic STT coded system.

and systematic STT code, as shown in Figure 1. The transmitted source bits are convolutionally encoded and directed to a random channel interleaver $\pi_{c}$. The convolutional encoder denoted as $\mathrm{CC}(2,1,5)$ is a $\frac{1}{2}$-rate Recursive Systematic Convolutional (RSC) code having a constraint length of $K=5$ and octal generator polynomials of $G_{0}=35$ and $G_{1}=23$. The RSC codeword consists of a systematic information bit and a parity bit. Subsequently, the encoded bits are passed to a

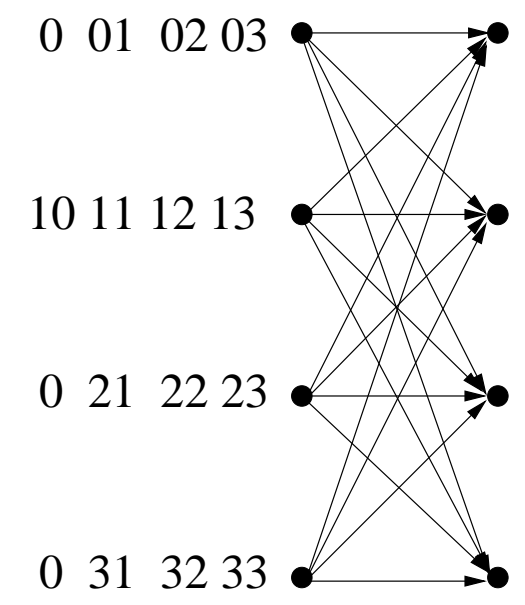

Figure 2: Trellis diagram of the systematic 4-state, 4PSK STT code [4].

systematic STT encoder using two transmit antennas, which is illustrated in Figure 2. The systematic STT encoder is referred to as $\operatorname{SSTTC}(n=4, m=4)$, since it is a $n=4$-state, $m=4$-PSK STT code. Upon receiving an input symbol, the SSTTC produces a symbol in each transmitter arm. These symbols are displayed at the side of each trellis state in Figure 2, which can be treated as the STT codeword, consisting of a systematic information symbol and a parity symbol. Note that we have employed the simple $\operatorname{SSTTC}(4,4)$ scheme instead of more complex systematic STT codes, since our aim was to highlight the turbo equalization principle. An example of a more complex systematic STT code is the $\operatorname{SSTTC}(8,8)$ scheme of reference [4]. The STT encoded symbols are interleaved by a random STT interleaver represented as $\pi_{s}$.

In our investigations, the transmission burst struc- ture consists of 100 data symbols. A two-path, symbolspaced fading Channel Impulse Response (CIR) of equal weights was used, where the Rayleigh fading statistics obeyed a normalised Doppler frequency of $3.3615 \times$ $10^{-5}$. The fading magnitude and phase was kept constant for the duration of a transmission burst, a condition which we refer to as employing burst-invariant fading. Furthermore, in order to investigate the lower bound performance of these systems, we have assumed that the CIR was perfectly estimated at the receiver. The Log-MAP algorithm [6] was employed in the channel equalizer, in the systematic STT decoder and in the RSC convolutional decoder.

\section{PRINCIPLE OF TURBO EQUALIZATION}

Let us now describe the turbo equalization principle in the context of the CC-SSTTC system. A vital rule for turbo equalizers is that the input information of a particular stage in the current iteration must not include the information contributed by that particular stage from the previous iteration, since then the information used in consecutive iterations would be dependent on each other [7]. The choice of convolutional codes and STT codes was constrained to the systematic variant of both codes. This is essential when calculating the extrinsic information of the STT decoder and that of the convolutional decoder.

We will elaborate on this issue by using the channel equalizer of Figure 3 as our first example. Note that the Log Likelihood Ratios (LLRs) are expressed in the vectorial form of [7], but using different actual notations. Here, the notations $G$ and $F$ are used to represent the LLRs of the systematic and parity symbols of the STT codeword, respectively, while $B$ and $A$ refers to the LLRs of the systematic and parity bits of the convolutional codeword. The superscript denotes the nature of the LLR, namely ' $a$ ' denotes the a priori information, ' $c$ ' is used for the composite a posteriori [8] information, ' $i$ ' for the combined channel and extrinsic [8] information and ' $e$ ' for the extrinsic [8] information. These definitions will be further augmented during our forthcoming discourse. Furthermore, the subscripts are used to represent the iteration index, while the argument within the brackets ( ) indicates the index of the receiver stage, where the equalizers are denoted as stage 0 , the STT decoder as stage 1 and the channel decoder as stage 2 . In the figures, we have also marked the connected points using circled bold notations.

In the first iteration at $p=1$, the channel equalizer of Figure 3 only evaluates the received signal $r(t)$, since there is no a priori feedback information, i.e. $F_{1}^{a}(0)=$ 0 and $G_{1}^{a}(0)=0$. Using the Log-MAP algorithm, the channel equalizer of Figure 3 computes the a posteri- 


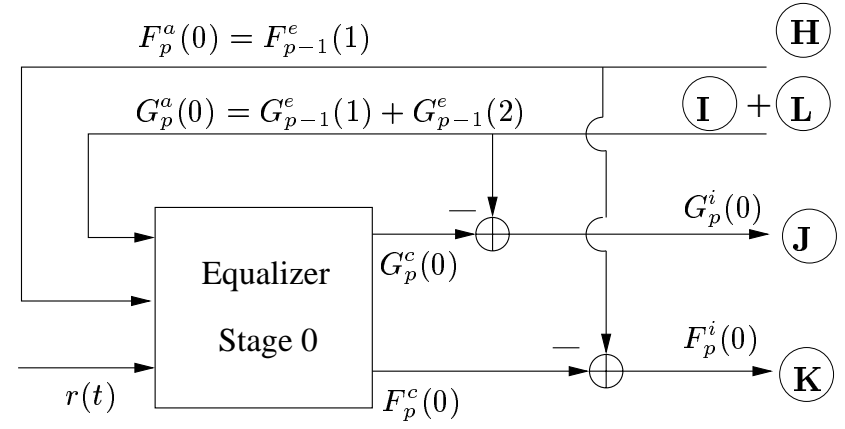

Figure 3: Schematic of the channel equalizer showing the received LLRs and the LLRs generated.

ori LLR values $G_{1}^{c}(0)$ of the systematic STT coded symbol and the a posteriori LLRs $F_{1}^{c}(0)$ of the parity symbols. Subsequently, these LLR values are deinterleaved by the STT deinterleaver $\pi_{s}^{-1}$ - which is not explicitly shown in the figures - and passed to the $\operatorname{SSTTC}(4,4)$ decoder of Figure 4. However, in the subsequent iterations, the channel equalizer will receive the additional a priori information $G_{p}^{a}(0)$ and $F_{p}^{a}(0)$ concerning the STT codeword - consisting of the systematic and parity symbols - from the other decoding stages. In order to avoid passing the a priori information contributed by the other concatenated decoding stages back to these stages, we subtract the a priori LLRs $F_{p}^{a}(0)$ and $G_{p}^{a}(0)$ from the corresponding a posteriori LLRs $F_{p}^{c}(0)$ and $G_{p}^{c}(0)$ in order to derive the combined channel and extrinsic information $F_{p}^{i}(0)$ and $G_{p}^{i}(0)$, as shown in Figure 3 .

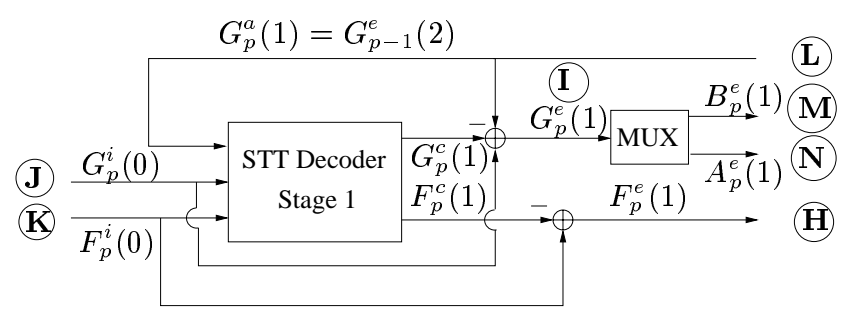

Figure 4: Schematic of the systematic STT decoder showing the input and output LLRs.

Let us now consider the operation of the STT decoder in Figure 4. The decoder receives the combined channel and the extrinsic LLRs $F_{p}^{i}(0)$ and $G_{p}^{i}(0)$ produced by the channel equalizer of Figure 3 in the current $p$ th iteration and the a priori LLRs $G_{p}^{a}(1)$ of the systematic symbol, which is the extrinsic LLR $G_{p-1}^{e}(2)$ generated by the channel decoder of Figure 5 during the previous turbo equalization iteration, namely iteration $p-1$. Using these LLR values, the STT decoder computes the a posteriori LLRs $G_{p}^{c}(1)$ and $F_{p}^{c}(1)$ of the STT coded systematic and parity symbols, respectively. As depicted in Figure 4, the a priori LLRs $G_{p}^{a}(1)$ and the combined channel and extrinsic LLRs $G_{p}^{i}(0)$ corresponding to the systematic symbol are subtracted from the composite a posteriori $\operatorname{LLRs} G_{p}^{c}(1)$, in order to obtain the extrinsic information $G_{p}^{e}(1)$ of the systematic symbol. By contrast, the extrinsic LLRs $F_{p}^{e}(1)$ of the space time coded 'parity' symbols are obtained by subtracting the combined channel and extrinsic LLRs $F_{p}^{i}(0)$ from the a posteriori LLRs $F_{p}^{c}(1)$ corresponding to the space-diversity related 'parity' symbols.

Note that if a non-systematic STT encoder were employed, the STT decoder would be unable to determine the extrinsic LLRs by subtracting the a priori LLRs, which constitute the LLRs of the systematic component of the STT codeword, from the a posteriori LLRs. This is because the a posteriori LLRs of the STT code do not correspond to the systematic STT coded symbol. Specifically, they correspond to the diversity-related STT coded 'parity' symbol. Therefore, unless the STT code were systematic, the a priori information $G_{p}^{a}(1)=$ $G_{p-1}^{e}(2)$ produced by the convolutional decoder could not be removed, potentially allowing this information to be fed back into the convolutional decoder. Hence, it is important that the STT encoder used is systematic.

Subsequently, these extrinsic LLRs are then passed to the channel deinterleaver, which is not explicitly shown in the figures. The extrinsic LLRs $F_{p}^{e}(1)$ and $G_{p}^{e}(1)$ of the STT codeword seen in Figure 4 become a priori LLRs of the channel equalizer, while the extrinsic LLRs $B_{p}^{e}(1)$ corresponding to the channel encoded systematic bits are extracted from $G_{p}^{e}(1)$ to become the a priori LLRs of the convolutional decoder. Note that the extrinsic LLRs $A_{p}^{e}(1)$ referring to the channel encoded parity bits are not used in the channel decoding operation, since the a priori information corresponding to the decoder trellis transition probability is only influenced by the systematic bit, but not by the parity bit.

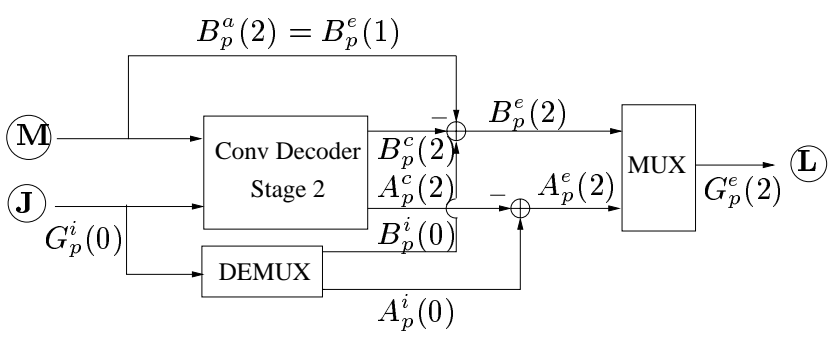

Figure 5: Schematic of the systematic convolutional decoder depicting the input and output LLRs.

As for the convolutional decoder depicted in Figure 5 , two inputs are received, namely the a priori LLRs 
$B_{p}^{a}(2)$ concerning the systematic bit of the convolutional codeword and the combined channel and extrinsic LLRs $G_{p}^{i}(0)$ of the systematic STT coded symbol generated by the channel equalizer of Figure 3, which consists of the convolutional coded systematic and parity bits. Using these LLR values, the decoder computes the a posteriori LLRs $A_{p}^{c}(2)$ and $B_{p}^{c}(2)$ of the codeword, as seen in Figure 5. In order to determine the extrinsic LLRs $B_{p}^{e}(2)$ of the systematic bits, the a priori LLRs $B_{p}^{a}(2)$ and the combined channel and extrinsic LLRs $B_{p}^{i}(0)$ corresponding to the systematic bit are subtracted from the a posteriori LLRs $B_{p}^{c}(2)$ related to the systematic bits, as shown in Figure 5. By contrast, the extrinsic LLRs $A_{p}^{e}(2)$ of the parity bits are extracted by subtracting the combined channel and extrinsic LLRs $A_{p}^{i}(0)$ from the a posteriori LLRs $A_{p}^{c}(2)$ corresponding to the parity bits. For the same reasons as mentioned above, upon using a non-systematic convolutional decoder, the a priori information could not be removed from the a posteriori information. Consequently, the a priori LLRs originating from the STT decoder would be inevitably passed back to the STT decoder in the following iteration. Therefore, it is important that systematic convolutional codes are employed in the turbo-equalized CC-SSTTC system. The extrinsic LLRs $B_{p}^{e}(2)$ and $A_{p}^{e}(2)$ of the systematic and parity bits, respectively, are combined, in order to form $G_{p}^{e}(2)$ and subsequently passed to the channel interleaver. The channel-deinterleaved extrinsic LLRs of the channel encoded bits are used as the a priori LLRs of the STT decoder in the next iteration. These extrinsic LLRs are also added to the extrinsic LLRs $G_{p}^{e}(1)$ of the STT decoder and subsequently combined with $F_{p}^{e}(1)$ generated by the STT decoder in order to form the a priori LLRs of the channel equalizer for the forthcoming $(p+1)$ th iteration.

\section{RESULTS AND DISCUSSION}

Let us now characterise the performance of the turboequalized CC-SSTTC system. Figure 6 shows the performance of the investigated system using various STT interleaving sizes, namely 100, 400, 800, 1600, 3200 and 6400 bits after eight turbo equalization iterations. It was observed in Figure 6 that by increasing the STT interleaving size from 100 to 6400, the interleaving gain attained was approximately $5.5 \mathrm{~dB}$ at BER $=10^{-4}$. Therefore, as expected, higher interleaving gains can be achieved by using longer STT interleavers. As our next performance metric, we defined the interleaver gain as the achievable $E_{b} / N_{o}$ reduction at $\mathrm{BER}=10^{-4}$ upon increasing the interleaver memory relative to a memory of 100 bits. The values of this metric were then extracted from Figure 6 and plotted in Figure 7. As illustrated in Figure 7, the interleaver gain gradually

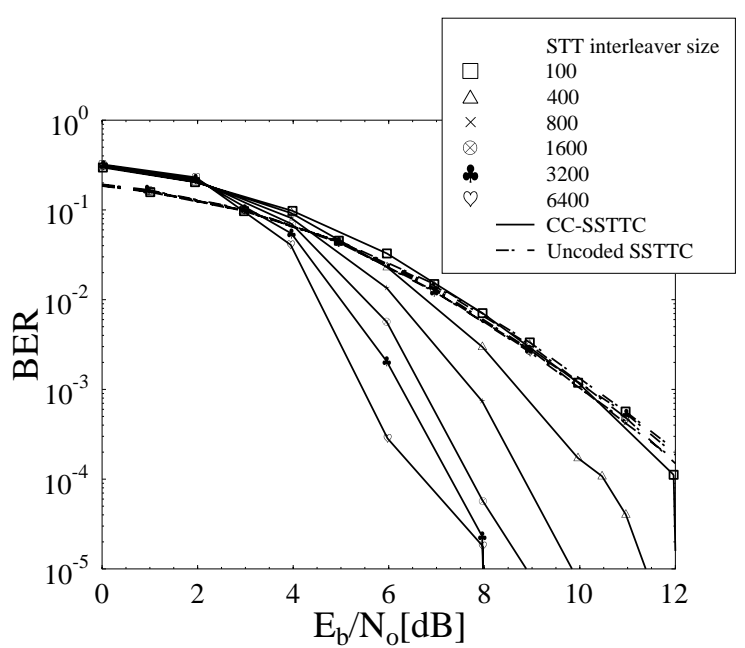

Figure 6: BER performance of the turbo-equalized serially concatenated convolutional coded and STT coded system using various STT interleaver sizes, namely 100, 400, 800, 1600, 3200 and 6400 bits, after eight turbo equalization iterations. The performance of the uncoded SSTTC system is also shown as a benchmark. The throughput of the CC-SSTTC system is 1 bits/symbol (BPS), while the uncoded SSTTC system has a BPS throughput of 2 .

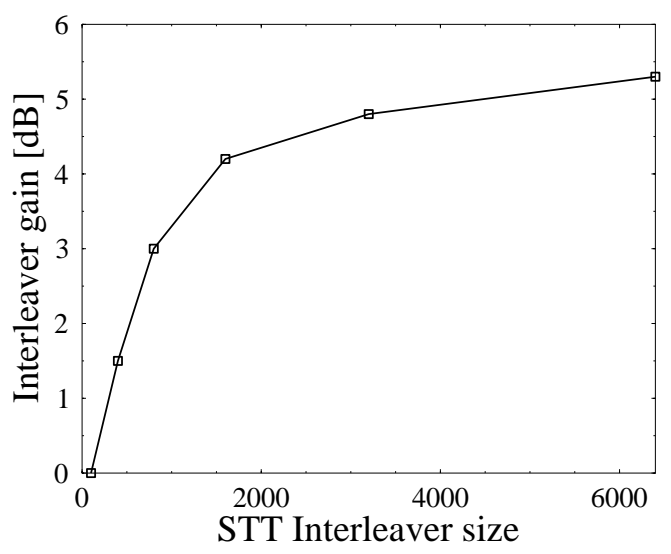

Figure 7: Interleaver gain achieved by the CC-SSTTC system at BER $=10^{-4}$ for STT interleaver sizes of 100 , 400, 800, 1600, 3200 and 6400 bits. 
saturates, when the STT interleaver size is in excess of 1600 bits. When the BER performance of the turbo-

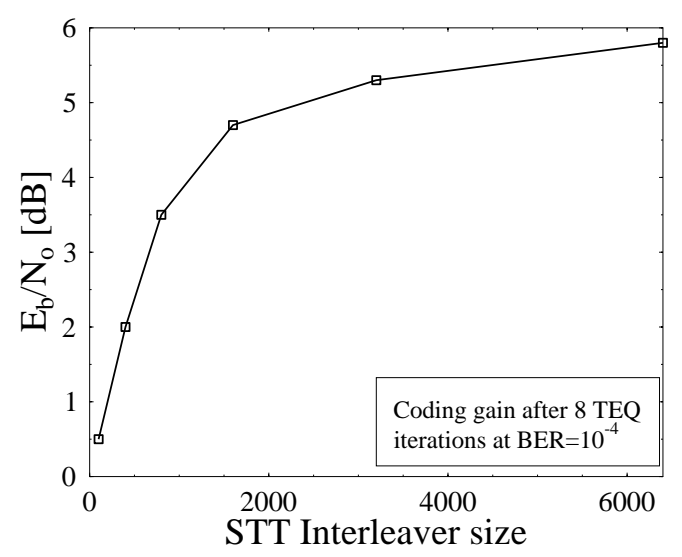

Figure 8: Coding gain achieved by the CC-SSTTC system over the uncoded $\operatorname{SSTTC}(4,4)$ system at BER = $10^{-4}$ for STT interleaver sizes of $100,400,800,1600$, 3200 and 6400 bits. The throughput of the CC-SSTTC system is $1 \mathrm{bits} / \mathrm{symbol}$ (BPS), while the uncoded SSTTC system has a BPS throughput of 2 .

equalized CC-SSTTC system is compared to that of the STT coded scheme using no channel coding, as expected, the channel coded system outperformed the uncoded scheme. It was observed from Figure 8 that a coding gain of approximately $5.7 \mathrm{~dB}$ was achieved by the CC-SSTTC system using a 6400-bit STT interleaver, when compared to an unprotected STT coded system at $\mathrm{BER}=10^{-4}$.

\section{CONCLUSIONS}

A turbo equalization scheme was proposed for a serially concatenated systematic convolutional coded and systematic STT coded system. It was observed in Figure 6 that higher interleaving gains were achieved for longer STT interleavers. By using a 6400-bit STT interleaver, an interleaving gain of approximately $5.5 \mathrm{~dB}$ was obtained, when compared to the CC-SSTTC scheme using a 100-bit STT interleaver. Figure 7 showed that the interleaving gain saturated for STT interleaver sizes in excess of 1600 bits. Finally, the performance of the CC-SSTTC system was compared to the uncoded STT coded system, in order to determine the corresponding coding gain. It was observed from Figure 8 that a coding gain of approximately $5.7 \mathrm{~dB}$ was achieved by the CC-SSTTC system using a 6400-bit STT interleaver, when compared to the STT coded system using no channel coding at BER $=10^{-4}$. Hence, it can be concluded that further BER improvements can be achieved in jointly detected channel coded, turboequalized STT coded systems.

\section{Acknowledgement}

This work has been funded in the framework of the IST project IST-1999-12070 TRUST, which is partly funded by the European Union. The authors would like to acknowledge the contributions of their colleagues.

\section{REFERENCES}

[1] T. Ojanperä and R. Prasad, Wideband CDMA for Third Generation Mobile Communications. Artech House, 1998.

[2] C. Berrou, A. Glavieux, and P. Thitimajshima, "Near Shannon Limit Error-Correcting Coding and Decoding: Turbo Codes," in Proceedings of the International Conference on Communications, (Geneva, Switzerland), pp. 1064-1070, 2326 May 1993.

[3] C. Douillard, A. Picart, M. Jézéquel, P. Didier, C. Berrou, and A. Glavieux, "Iterative correction of intersymbol interference: Turbo-equalization," European Transactions on Communications, vol. 6, pp. 507-511, September-October 1995.

[4] V. Tarokh, N. Seshadri, and A. R. Calderbank, "Space-Time Codes for High Data Rate Wireless Communication: Performance Criterion and Code Construction," IEEE Transactions on Information Theory, vol. 44, pp. 744-765, March 1998.

[5] G. Bauch, A. F. Naguib, and N. Seshadri, "MAP Equalization of Space-time Coded Signals over Frequency Selective Channels," in Proceedings of the Wireless Communications and Networking Confeerence, (New Orleans, USA), September 1999 .

[6] P. Robertson, E. Villebrun, and P. Hoeher, "A Comparison of Optimal and Sub-Optimal MAP Decoding Algorithms Operating in the Log Domain," in Proceedings of the International Conference on Communications, (Seattle, United States), pp. 1009-1013, 18-22 June 1995.

[7] M. J. Gertsman and J. L. Lodge, "Symbol-by-symbol MAP demodulation of CPM and PSK signals on Rayleigh flatfading channels," IEEE Transactions on Communications, vol. 45, pp. 788-799, July 1997.

[8] L. R. Bahl, J. Cocke, F. Jelinek, and J. Raviv, "Optimal Decoding of Linear Codes for Minimising Symbol Error Rate," IEEE Transactions on Information Theory, pp. 284-287, March 1974. 